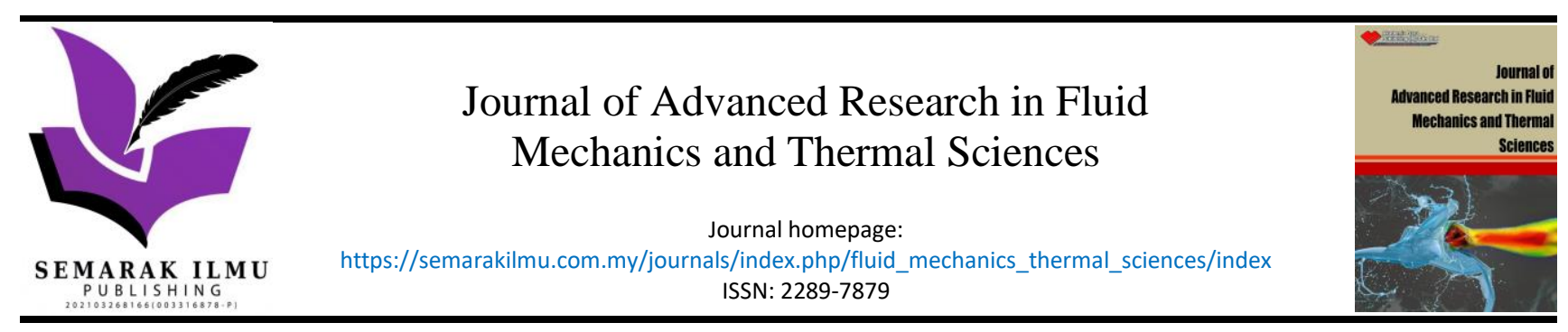

\title{
Homotopy Analysis of Carreau Fluid Flow Over a Stretching Cylinder
}

\author{
Lim Yeou Jiann ${ }^{1}$, Sharidan Shafie ${ }^{1,},{ }^{*}$ Ahmad Qushairi Mohamad ${ }^{1}$, Noraihan Afiqah Rawi $^{1}$ \\ 1 Department of Mathematical Sciences, Faculty Sciences, Universiti Teknologi Malaysia, Skudai, 81310 Johor Bahru, Johor, Malaysia
}

\section{ARTICLE INFO ABSTRACT}

\section{Article history:}

Received 26 April 2021

Received in revised form 20 August 2021

Accepted 3 September 2021

Available online 29 October 2021

\section{Keywords:}

Homotopy Analysis; Carreau Fluid

Flow; Stretching Cylinder

\begin{abstract}
Carreau fluid flows past a stretching cylinder is elucidated in the present study. The transformed self-similarity and dimensionless boundary layer equations are solved by using the Homotopy analysis method. A convergence study of the method is illustrated explicitly. Series solutions of the highly nonlinear differential equations are computed and it is very efficient in demonstrating the characteristic of the Carreau fluid. Validation of the series solutions is achieved via comparing with earlier published results. Those results are obtained by using the Keller-Box method. The effects of the Weissenberg number and curvature parameter on the velocity profiles are discussed by graphs and tabular. The velocity curves have shown different behavior in $n<1$ and $n \geq 1$ for an increase of the Weissenberg number. Further, the curvature parameter $K$ does increase the velocity profiles.
\end{abstract}

\section{Introduction}

Flow over a stretching cylinder has relevance in a number of the industrial manufacturing process such as annealing and tinning of copper cords, fabrication of papers, and glass fiber production. Two-dimensional flow is generally utilized to depict the flow over a cylinder that has a body radius greater than the boundary layer thickness. However, the flow may be acknowledged as axisymmetric when the radius of the cylinder is in the same order as the boundary layer thickness. In this particular case, a transverse curvature term is introduced in the governing equations which may have an impact on the velocity profiles. This is essential in certain industrial applications such as food processing, polymer, blood flow in a microcirculatory system, and wire drawing where an accurate prediction of flow is needed so that a sufficient boundary layer can occur on slender or near slender bodies. In view of this, Wang [1] conducted a study to discuss the steady flow of an incompressible and viscous fluid over a stretching cylinder. An exact similarity solution of the governing equations was obtained for the stretching surface. Motivated by this work, Ishak et al., [2] extended the study by including the effect of a magnetic field. The flow and heat transfer of the fluid outside of the stretching tube was determined numerically using the Keller-Box method.

\footnotetext{
* Corresponding author.

E-mail address: sharidan@utm.my

https://doi.org/10.37934/arfmts.88.2.8092
} 
Further, Ishak et al., [3] considered the effects of suction and injection on the stretching permeable cylinder. The numerical solutions have shown that skin friction is reduced by the injection parameter. Besides, Lok et al., [4] have elucidated steady mixed convection flow near an axisymmetric stagnation point on stretching or shrinking vertical cylinder. The boundary layer flow is driven by a stretching cylinder in the influence of variable thermal conductivity has been discussed by Rangi and Naseem [5]. The Keller-box technique was practiced and found that the curvature of the cylinder has a significant influence on the flow and temperature field. Vajravelu et al., [6] acknowledged the effect of the transverse curvature, internal heat source, and temperaturedependent thermal conductivity on the axisymmetric MHD flow and heat transfer through a nonisothermal stretching cylinder. Numerical results for two different types of non-isothermal boundary conditions were calculated by the authors. Mukhopadhyay [7] numerically analyzed the effect of the porous medium on the mixed convection flow along with a vertical stretching cylinder. Then, Mukhopadhyay [8] extended the study by considering the boundary layer flow of a fluid and heat transfer towards a stretching cylinder under the effect of magnetic field and partial slip boundary condition. The numerical results depicted that the velocity decreases but the temperature increases as the magnetic and slip parameters were increased. Adnan et al., [9] investigated the fluid flow over a permeable stretching or shrinking cylinder near a stagnation point. The author had analyzed the stability of the dual numerical solutions of the problem. Most of the above-mentioned studies are focused on the Newtonian fluid.

Newtonian fluids are defined as fluid where the viscosity changes are independent of the shear rate. However, in many practical applications, the fluid is non-Newtonian such as pharmaceutical chemicals, jams, polymer fluids, China clay, animal blood, synthetic lubricants, and paints. The viscosity of those fluids is dependent on the shear rate and this is important when dealing with lubrication problems and polymer processing. Accordingly, various constitutive models for nonNewtonian fluid have been presented in the literature regarding the diversity of the flow in nature such as the power-law model, Casson fluid, Ellis fluid, and cross fluid [10]. The effect of the Arrhenius activation energy, thermal radiation, and film thickness on a viscoelastic thin film nanofluid over a stretching cylinder and generation of the entropy was studied by Usman et al., [11] by using the homotopy analysis method. The power-law constitutive relation is the simplest model but it provides inaccuracy to predict the viscosity when the shear rate is very small or very large. In an assessment of such limitation, Carreau [12] proposed a rheological model which is a combination of power-law models and Newtonian fluid. It gives a finite viscosity at a low or high shear rate. The Carreau fluid has gained the attention of plenty of researchers due to its significance in chemical engineering and polymeric suspensions. Tshehla [13] investigated the Carreau fluid flow over an inclined plane with a free surface. The profiles of the velocity and temperature were derived using the asymptotic technique and numerical method and the influences by the viscosity variation parameter, the Biot, and the Brinkman numbers were discussed.

Olajuwon [14] established a numerical investigation on the effect of the thermal radiation and thermal diffusion on the heat and mass transport in an MHD Carreau fluid past a vertical porous plate. The authors found that the power index and material parameters have caused an increment in the fluid velocity. Further, Khan et al., [15] applied the Runge-Kutta Fehlberg method along with the shooting technique to analyze the Carreau fluid flow and heat transfer over a non-linear stretching sheet. Hayat et al., [16] elucidated a two-dimensional Carreau fluid flow toward a permeable convective heated stretching sheet. Analytic solutions of the governing equations were computed by utilizing the homotopy analysis method (HAM). The effect of the embedded parameters such as power-law index, relaxation time, and Biot number on the velocity and 
temperature profiles was discussed. Khan et al., [17] scrutinized the boundary layer flow and heat transfer of three-dimensional Carreau fluid over a bidirectional stretching sheet under the effect of non-linear thermal radiation. A comparison study between bvp4c results with the analytical results determined by the HAM was conducted to analyze the legitimacy of numerical results. A conflict behavior was observed by the authors for the shear-thinning fluid and shear thickening fluid on the velocity profile for the various value of the Weissenberg number. More recent research on the Carreau fluid can be found in [18-20].

Recently, Salahuddin [21] studied the Carreau fluid flow over a stretching cylinder. The similarity solutions of the governing equations were obtained by the Keller box and shooting method. The influences of the different values of the Weissenberg number, curvature parameter, and power-law index on the velocity distribution were analyzed. However, in the study, the case for the shearthinning fluid is not discussed. Therefore, the objective of the present investigation is to reproduce the work done by Salahuddin [21] by including the case of shear-thinning fluid before going to explore the heat and mass transfer in the fluid. The homotopy analysis method is applied to solve the problem due to the effectiveness of the method in solving highly non-linear differential equations. Plenty of researches have successfully applied the method to solve the complicated nonlinear fluid problem as seen in [18, 19, 22-25]. HAM was first introduced by Shijun Liao in 1992 when he is doing his Ph.D. dissertation at Shanghai Jiaotong University [26]. The details of the methods can be found in [24], [25], and [27].

\section{Mathematical Formulation}

A steady two-dimensional and incompressible Carreau fluid flow over a permeable stretching cylinder is considered in the present research. As depicted in Figure 1, the x-axis is measured along the axis of the cylinder, and the $r$-axis is taken in the radial direction.

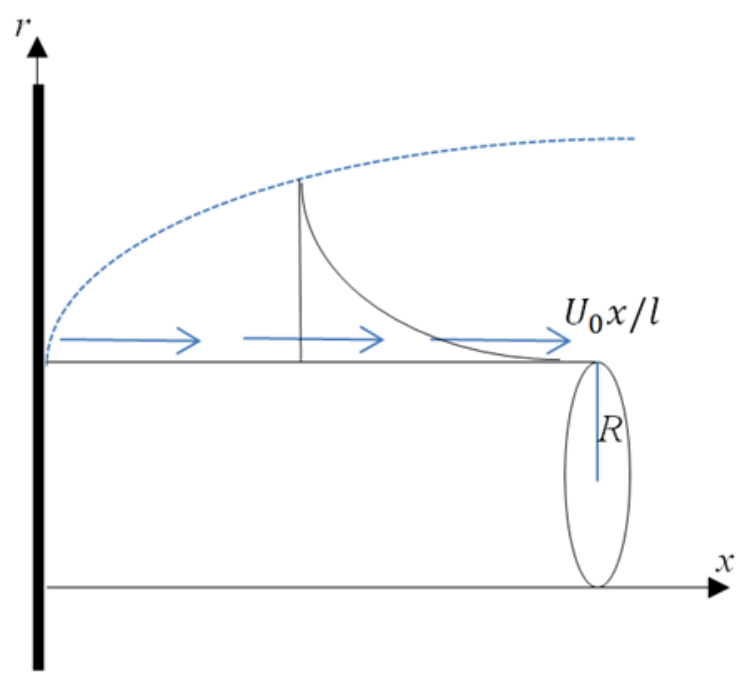

Fig. 1. Schematic geometry of the problem

The Cauchy stress tensor for Carreau fluid is given by $[15,21,28]$.

$$
\tau_{i j}=\mu \mathbf{A}_{1},
$$

With 


$$
\frac{\mu-\mu_{\infty}}{\mu_{0}-\mu_{\infty}}=\left[1+(\Gamma \dot{\gamma})^{2}\right]^{\frac{n-1}{2}}
$$

where $\mathbf{A}_{\mathbf{1}}$ is the first kind Rivlin-Erickson tensor, $\mu$ is the shear rate viscosity, $\mu_{0}$ is the zero-shearrate viscosity, $\mu_{\infty}$ is the infinite-shear-rate viscosity, $\Gamma$ is a material time constant, and $n$ is the power-law index. $\dot{\gamma}$ is the shear rate and is expressed as

$$
\dot{\gamma}=\sqrt{\frac{1}{2} \sum_{i} \sum_{j} \dot{\gamma}_{i j} \dot{\gamma}_{i j}}=\sqrt{\frac{1}{2} \Pi}=\sqrt{\frac{1}{2} \operatorname{tr}\left(A_{1}^{2}\right)},
$$

where $\Pi$ is the second invariant strain tensor. We consider in the constitutive Eq. (1), the case for which $\eta_{\infty}=0$ and $\Gamma \dot{\gamma}<1$, thus by binomial expansion, Eq. (2) become

$$
\mu=\mu_{0}\left[1+\frac{n-1}{2}(\Gamma \dot{\gamma})^{2}\right]
$$

Substitute Eq. (4) into (1), the stress tensor can be written as

$$
\tau_{i j}=\mu_{0}\left[1+\frac{n-1}{2}(\Gamma \dot{\gamma})^{2}\right] \mathbf{A}_{1}
$$

The boundary layer approximation of the continuity and momentum equations of the Carreau fluid flow is represented as

$$
\begin{aligned}
& \frac{\partial(r u)}{\partial x}+\frac{\partial(r v)}{\partial x}=0 \\
& u \frac{\partial u}{\partial x}+v \frac{\partial u}{\partial r}=v\left(\frac{\partial^{2} u}{\partial r^{2}}+\frac{1}{r} \frac{\partial u}{\partial r}+\frac{3 \Gamma^{2}(n-1)}{2}\left(\frac{\partial u}{\partial r}\right)^{2} \frac{\partial^{2} u}{\partial r^{2}}+\frac{\Gamma^{2}(n-1)}{2 r}\left(\frac{\partial u}{\partial r}\right)^{3}\right)
\end{aligned}
$$

where $v$ is the kinematic viscosity. The corresponding boundary conditions are

$$
\begin{aligned}
& u=\frac{U_{0} x}{l}, \quad v=0, \quad \text { at } \quad r=R, \\
& u \rightarrow 0, \quad \text { as } \quad r \rightarrow \infty,
\end{aligned}
$$

where $U_{0}$ is a positive constant, $r$ and $x$ are the cylindrical polar coordinates which $r$ is in the radial direction and $x$ represents the axial direction, and $l$ is the characteristic length.

Following similarity, variables are introduced to reduce the partial differential equations (6)-(7) into the ordinary differential equations.

$$
\eta=\sqrt{\frac{U_{0}}{l v}}\left(\frac{r^{2}-R^{2}}{2 R}\right), \quad u=\frac{x U_{0}}{l} f^{\prime}, \quad v=-\frac{R}{r} \sqrt{\frac{v U_{o}}{l}} f .
$$

Substitute Eq. (9) into Eq. (6) and (7). Eq. (6) is automatically satisfied and Eq. (7) reduces to 


$$
\begin{aligned}
& \frac{3(n-1)}{2} W e^{2}(1+2 K \eta)\left(f^{\prime \prime}\right)^{2}\left(K f^{\prime \prime}+(1+2 K \eta) f^{\prime \prime \prime}\right)+2 K f^{\prime \prime} \\
& f f^{\prime \prime}-\left(f^{\prime}\right)^{2}+\frac{(n-1)}{2} W e^{2} K(1+2 K \eta)\left(f^{\prime \prime}\right)^{3}+(1+2 K \eta) f^{\prime \prime \prime}=0 .
\end{aligned}
$$

Utilizing the similarity variables in the boundary conditions (8), we have

$$
f(0)=0, \quad f^{\prime}(0)=1, \quad f^{\prime}(\infty)=0,
$$

where the prime denotes differentiation with respect to $\eta$, We are the Weissenberg number and $K$ is the curvature parameter which is represented as

$$
K=\frac{1}{R} \sqrt{\frac{v l}{U_{0}}}, \quad W e=\Gamma x \sqrt{\frac{U_{0}^{3}}{v l^{3}}}
$$

The physical quantity of interest skin friction coefficient $\left(\frac{1}{2} C_{f} R e_{x}^{1 / 2}\right)$ is expressed as

$$
\frac{C_{f} \operatorname{Re}_{x}^{1 / 2}}{2}=f^{\prime \prime}(0)+\frac{n-1}{2} W e^{2}\left[f^{\prime \prime}(0)\right]^{3}
$$

Where $R e_{x}^{1 / 2}=x \sqrt{\frac{U_{0}}{v l}}$.

\section{Solution by Homotopy Analysis Method}

In this section, the HAM is utilized to obtain the approximate analytical solutions of the Carreau fluid flows through a stretching cylinder. Eq. (14) and (15) are the initial guesses and the linear operator selected in the present study for the governing equations of the problem.

$f_{0}(\eta)=1-e^{-\eta}$,

$L_{f}=f^{\prime \prime \prime}+f^{\prime \prime}$

The above auxiliary linear operator has the following properties:

$L_{f}\left(c_{1}+c_{2} \eta+c_{3} e^{-\eta}\right)=0$

where $c_{i}, i=1,2,3$ are arbitrary constants. The zeroth-order deformation equation can be expressed as

$(1-p) L_{f}\left\{f(\eta: p)-f_{0}(\eta)\right\}=p \hbar_{f} N_{f}\{f(\eta: p)\}$ 


$$
\begin{aligned}
N_{f}\{f(\eta: p)\}= & \frac{3(n-1)}{2} W e^{2}(1+2 K \eta)\left[f^{\prime \prime}(\eta: p)\right]^{2}\left[K f^{\prime \prime}(\eta: p)+(1+2 K \eta) f^{\prime \prime \prime}(\eta: p)\right]+ \\
& (1+2 K \eta) f^{\prime \prime \prime}(\eta: p)+\frac{(n-1)}{2} W e^{2} K(1+2 K \eta)\left[f^{\prime \prime}(\eta: p)\right]^{3}+ \\
& 2 K f^{\prime \prime}(\eta: p)+f(\eta: p) f^{\prime \prime}(\eta: p)-\left[f^{\prime}(\eta: p)\right]^{2} .
\end{aligned}
$$

Where $p$ is an embedding parameter, $\hbar_{f}$ is the non-zero control convergence parameter and $N_{f}$ is the nonlinear operator. For $p=0$ and $p=1$, we have

$$
f(\eta: 0)=f_{0}(\eta), \quad f(\eta: 1)=f(\eta)
$$

As the embedding parameter $p$ approach 1 from $0, f(\eta: p)$ vary from initial guesses $f_{0}$ to the exact solution $f(\eta)[24,26]$. Taylor's series expansion at $\eta=0$ of the function $f(\eta: p)$ yields

$$
f(\eta: p)=f_{0}(\eta)+\sum_{k=1}^{\infty} f_{k}(\eta) p^{k}
$$

where

$$
f_{k}(\eta)=\left.\frac{1}{k !} \frac{\partial^{k} f(\eta: p)}{\partial p^{k}}\right|_{p=0}
$$

Series (20) is also called the Maclaurin series. On the assumption that the Eq. (20) is analytic in $p \epsilon[0,1]$ and converge at $p=1$ to $f(\eta)$, thus we have

$$
f(\eta)=f_{0}(\eta)+\sum_{k=1}^{\infty} f_{k}(\eta)
$$

$f_{k}$ is obtained by the so-called high-order deformation equations governed by the chosen auxiliary linear operator. Differentiating $k$-times the zeroth-order deformation of Eq. (17) with respect to $p$, and dividing then by $k$ ! and finally setting $p=0$, one has the $k$ th order deformation equations as

$$
L_{f}\left\{f_{k}(\eta)-\chi_{k} f_{k-1}(\eta)\right\}=\hbar_{f} R_{k-1}\left(N_{f}\{f(\eta: p)\}\right)
$$

where,

$$
\chi_{k}=\left\{\begin{array}{ll}
0, & k \leq 1, \\
1, & k>1,
\end{array} R_{k-1}=\left.\frac{1}{(k-1) !} \frac{\partial^{k-1}}{\partial p^{k-1}}\right|_{p=0},\right.
$$

and by using the Leibniz formula we have, 


$$
\begin{aligned}
R_{k-1}\left(N_{f}\{f(\eta: p)\}\right)= & \frac{3(n-1)}{2} W e^{2}(1+2 K \eta)\left[\sum_{i=0}^{k-1} f_{k-1-i}^{\prime \prime} K \sum_{j=0}^{i} f_{i-j}^{\prime \prime} f_{i}^{\prime \prime}\right]+ \\
& (1+2 K \eta)^{2} \frac{3(n-1)}{2} W e^{2}\left[\sum_{i=0}^{k-1} f_{k-1-i}^{\prime \prime} \sum_{j=0}^{i} f_{i-j}^{\prime \prime} f_{i}^{\prime \prime \prime}\right]+ \\
& \frac{(n-1)}{2} W e^{2} K(1+2 K \eta)\left[\sum_{i=0}^{k-1} f_{k-1-i}^{\prime \prime} K \sum_{j=0}^{i} f_{i-j}^{\prime \prime} f_{i}^{\prime \prime}\right]+ \\
& (1+2 K \eta) f_{k-1}^{\prime \prime \prime}+2 K f_{k-1}^{\prime \prime}+\left[\sum_{i=0}^{k-1} f_{k-1-i} f_{i}^{\prime \prime}\right]-\left[\sum_{i=0}^{k-1} f_{k-1-i}^{\prime} f_{i}^{\prime}\right] .
\end{aligned}
$$

It is worth mentioning that $f_{k}$ for $k \geq 1$ is governed by the linear operator (15) subjected to the linear boundary conditions that originate from the problem, which the solutions can be easily determined by using symbolic computation software such as Mathematica and Maple. Here, the developed Mathematica package BVPh 2.0 by Liao [25] has been utilized to compute the solutions. To determine the convergence control parameter $\hbar_{f}$, which has been used to guarantee the convergence of the series solution (22), the so-called average residual error technique at $k$ th-order approximation is applied in BVPh 2.0, defined by

$$
E_{k}^{f}\left(\hbar_{f}\right)=\frac{1}{Z+1} \sum_{i=0}^{Z}\left[N_{f}\left(\sum_{j=0}^{k} f_{j}\left(\eta_{i}\right)\right)\right]^{2} .
$$

where $Z$ is an integer and $\eta_{i}=i(\delta \eta), \delta \eta$ is the step size. At the $k$ th-order approximation, the optimal values of the convergence control parameter $\hbar_{f}$ is calculated by the minimum of the error $E_{k}^{f}\left(\hbar_{f}\right)$. The details can be found in the monograph of "Homotopy analysis method in nonlinear differential equations" by Liao [25]

\section{Results and Discussion}

Table 1 illustrates the optimal convergence control parameter for the case $K=0.2, W e=0.2$ and $n=1.2$, from first order up to the 6th- order of approximations. The table depicts that the error decreases to $5.08169 \times 10^{-5}$ by having the corresponding optimal convergence-control parameter $\hbar_{f}=-1.39028$ for the 6 th order of approximations. It is observed that the series solutions converge in the region of $-0.91316 \leq \hbar_{f} \leq-1.39028$. Since the homotopy analysis technique has provided great freedom in choosing the convergence control parameter value to adjust and control the convergence region of the series solutions. For generality, the following solutions in the present study are computed by using $\hbar_{f}=-1.19962$. In this way, the error distribution and profile of the series solution versus the order of approximations for the case $K=0.2, W e=0.2$, and $n=1.2$ are shown in Table 2 and Figure 2 respectively. The residual error is indeed reduced by increasing the order of approximation as seen in Figure 2 . 


\section{Table 1}

Optimal value for convergence-control parameter at different orders of approximation for $K=0.2, W e=0.2$ and $n=1.2$ with the 1 st type of linear operator

\begin{tabular}{lll}
\hline$k$ (order of approximation) & $E_{k}^{f}$ & $\hbar_{f}$ \\
\hline 1 & $2.17362 \times 10^{-4}$ & -0.91316 \\
3 & $9.42131 \times 10^{-5}$ & -1.19962 \\
6 & $5.08169 \times 10^{-5}$ & -1.39028 \\
\hline
\end{tabular}

\section{Table 2}

Square residual errors at different orders of the series solutions for $K=0.2, W e=0.2$ and $n=1.2$ and the convergence-control parameter $\hbar_{f}=-1.19605$ with the 1 st type of linear operator

\begin{tabular}{lll}
\hline$k$ (order of approximation) & $E_{k}^{f}$ & CPU time $(\mathrm{s})$ \\
\hline 10 & $3.78124 \times 10^{-5}$ & 70.801 \\
20 & $2.22196 \times 10^{-5}$ & 1234.34 \\
30 & $1.63594 \times 10^{-5}$ & 10541.4 \\
\hline
\end{tabular}

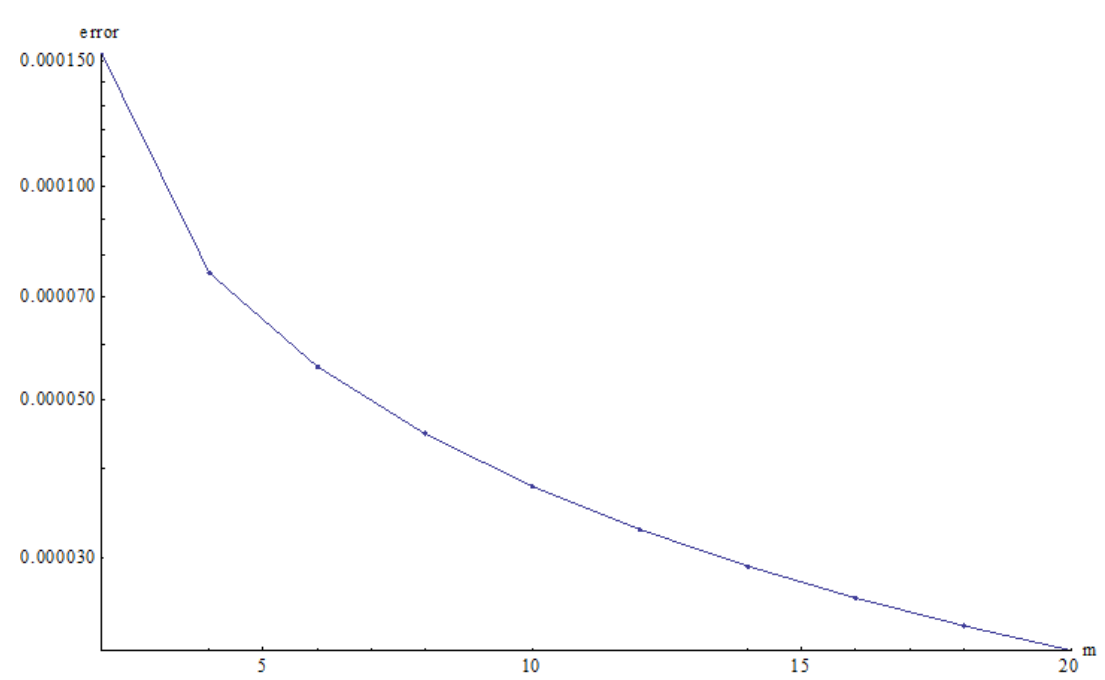

Fig. 2. Error profile of $f$, taking $K=0.2, W e=0.2$ and $n=1.2$ and the convergence-control parameter $\hbar_{f}=-1.19605$

Besides, another type of linear operator such as $L_{f}=f^{\prime \prime \prime}-f^{\prime}$, is choosing to determine the series solutions. Regarding the frame of HAM, one has great freedom to choose the auxiliary linear operator. Table 3 and 4 have depicted the residual error when computing the optimal convergence control parameter and at different orders of approximation of the series solutions. It is found that the computing time is lesser but the residual error for the second choice of a linear operator is larger as comparing Table 2 and Table 4. Further, the error is increased as the order of the approximations increase as seen in Table 4. Thus, the linear operator (15) is applied to generate the series solutions in this research.

\section{Table 3}

Optimal value for convergence-control parameter at different orders of approximation for $K=0.2, W e=0.2$ and $n=1.2$ with the 2nd type of linear operator

\begin{tabular}{lll}
\hline$k$ (order of approximation) & $E_{k}^{f}$ & $\hbar_{f}$ \\
\hline 1 & $2.31926 \times 10^{-4}$ & -1.11977 \\
3 & $1.26672 \times 10^{-4}$ & -1.45384 \\
6 & $7.01766 \times 10^{-5}$ & -1.66151 \\
\hline
\end{tabular}




\section{Table 4}

Square residual errors at different orders of the series solutions for $K=0.2$, We $=0.2$ and $n=1.2$ and the convergence-control parameter $\hbar_{f}=-2.02125$ with the $2 n d$ type of linear operator

\begin{tabular}{lll}
\hline$k$ (order of approximation) & $E_{k}^{f}$ & CPU time $(\mathrm{s})$ \\
\hline 10 & $5.17627 \times 10^{-5}$ & 60.18 \\
20 & $3.00657 \times 10^{-5}$ & 971.73 \\
30 & $2.18917 \times 10^{-5}$ & 9865.27 \\
\hline
\end{tabular}

In Table 5, limiting cases have been considered and compared with the previous literature results of the skin friction coefficient $f^{\prime \prime}(0)$ for different values of $K$ by Rangi and Naseem [5] and Salahuddin [21]. This is aimed to validate the obtained analytical approximation solution. It is found that the present results are in good agreement with the previous numerical results. Table 6 displays the skin friction coefficient for the various value of Weissenberg number We, curvature parameter $K$, and power-law index $n$. The skin friction $\left|f^{\prime \prime}(0)\right|$ is increased when the value of parameters $K, n$, and We increase. An opposite characteristic is observed for the We when the power-law index $n=$ 0.5 is used (see Table 5).

\section{Table 5}

Comparison of the local skin friction coefficient for different values of curvature parameter $K$ when

\begin{tabular}{lllll} 
We $=0$ and $n=1$ & \\
\hline$K$ & Rangi and Naseem [5] & (Shooting Method) [21] & (Keller Box) [21] & HAM \\
\hline 0 & -1.0000 & -1.0000 & -1.0000 & -1.0000 \\
0.25 & -1.0944 & -1.0944 & -1.0944 & -1.0992 \\
0.5 & -1.1887 & -1.1887 & -1.1887 & -1.1962 \\
0.75 & -1.2818 & -1.2818 & -1.2818 & -1.2920 \\
1.0 & -1.4593 & -1.4593 & -1.4593 & -1.3864 \\
\hline
\end{tabular}

Table 6

Comparison of the local skin friction coefficient for different values of physical parameters

\begin{tabular}{llll}
\hline$K$ & $n$ & $W e$ & HAM \\
\hline 0.2 & 0.5 & 0.2 & -1.07811 \\
- & - & 0.4 & -1.06608 \\
- & - & 0.6 & -1.04258 \\
- & - & 0.8 & -0.95753 \\
\hline 0.0 & 1.2 & 0.2 & -1.00116 \\
0.2 & - & - & -1.08342 \\
0.4 & - & - & -1.16359 \\
0.6 & - & - & -1.24259 \\
\hline 0.2 & 1.2 & 0.4 & -1.08784 \\
- & - & 0.6 & -1.09497 \\
- & - & 0.8 & -1.10449 \\
\hline 0.2 & 1.4 & 0.2 & -1.08491 \\
- & 1.6 & - & -1.08638 \\
- & 1.8 & - & -1.08784 \\
\hline
\end{tabular}

The effect of the curvature parameter $K$ on the velocity profile is demonstrated in Figure 3. Since, the increase of the curvature parameter $K$ is caused by the decrease in radius of the cylinder, which consequently decreases the contact area of the fluid with the boundary and hence reduces the resistive force offered by the surface to the fluid and the velocity. Therefore, the velocity is 
enhanced for higher values of curvature parameter $K$ as depicted in Figure 3. Both the velocity and the boundary layer thickness increase with an increase in the power-law index $n$. This phenomenon is illustrated in Figure 4. Uplifting of the power-law index has enriched the space in the fluid, since the momentum boundary layer becomes thicker, which results in the velocity of the fluid increasing [29].



Fig. 3. Velocity profile $f^{\prime}(\eta)$ versus $\eta$ for various values of $K$

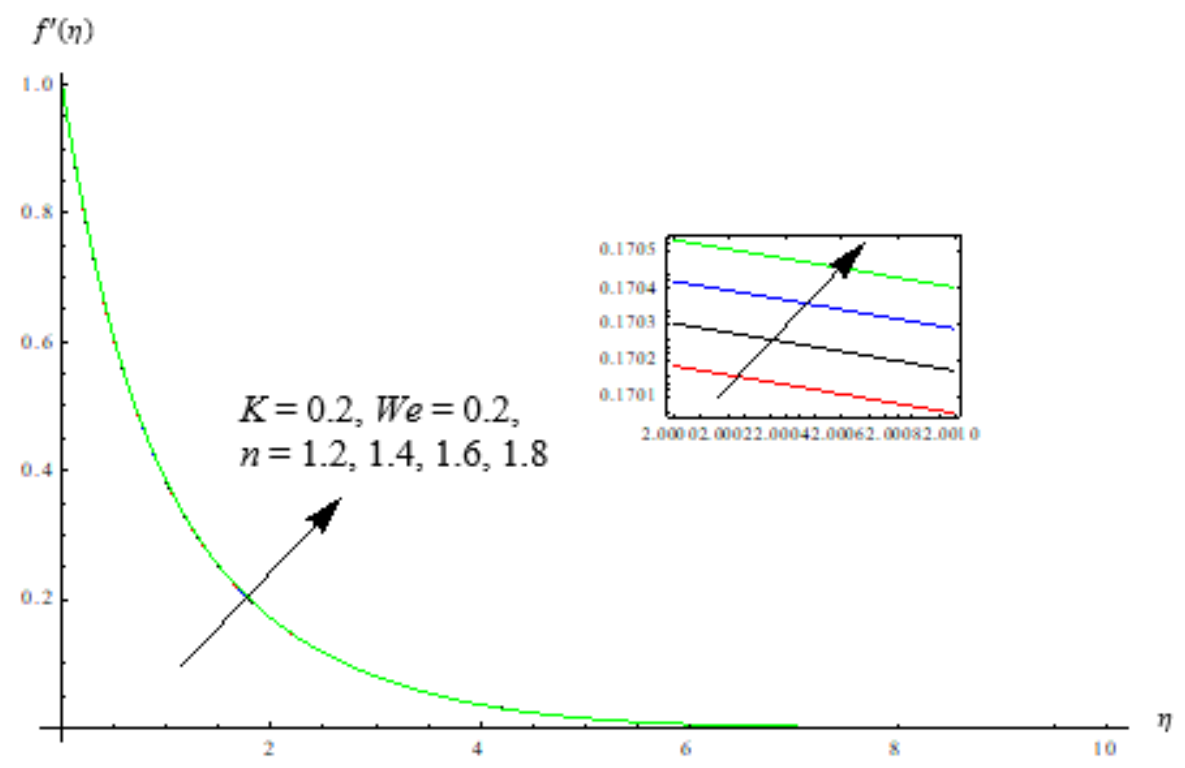

Fig. 4. Velocity profile $f^{\prime}(\eta)$ versus $\eta$ for various values of $n$

Figure 5 and 6 are plotted to perceive the behavior of the fluid profiles corresponding to the Weissenberg number We for $n>1$, the shear-thickening fluid, and $n<1$, the shear-thinning fluid respectively. Figure 5 clearly illustrates the velocity profile for the shear-thickening fluid is improved by increasing the values of Weissenberg number We. Physically, We are the relation of the relaxation time of the fluid as shown in Eq. (12). Thus, the relaxation time is increased as We increase. Accordingly, we assume $\Gamma \dot{\gamma}<1$, thus the shear rate decrease which results in low viscosity in the shear-thickening fluid. However, high viscosity is provided in the shear-thinning 
fluid. Subsequently, there is a decline in the velocity profile as demonstrated in Figure 6 . Notable, the results for $W e>1$ and $n=0.5$ are not converging, therefore the values $W e=0.2,0.4,0.6$, and 0.8 are used to compute the solutions as seen in Figure 6. More study is indeed needed to enlarge the understanding of the present problem and also the method applied.

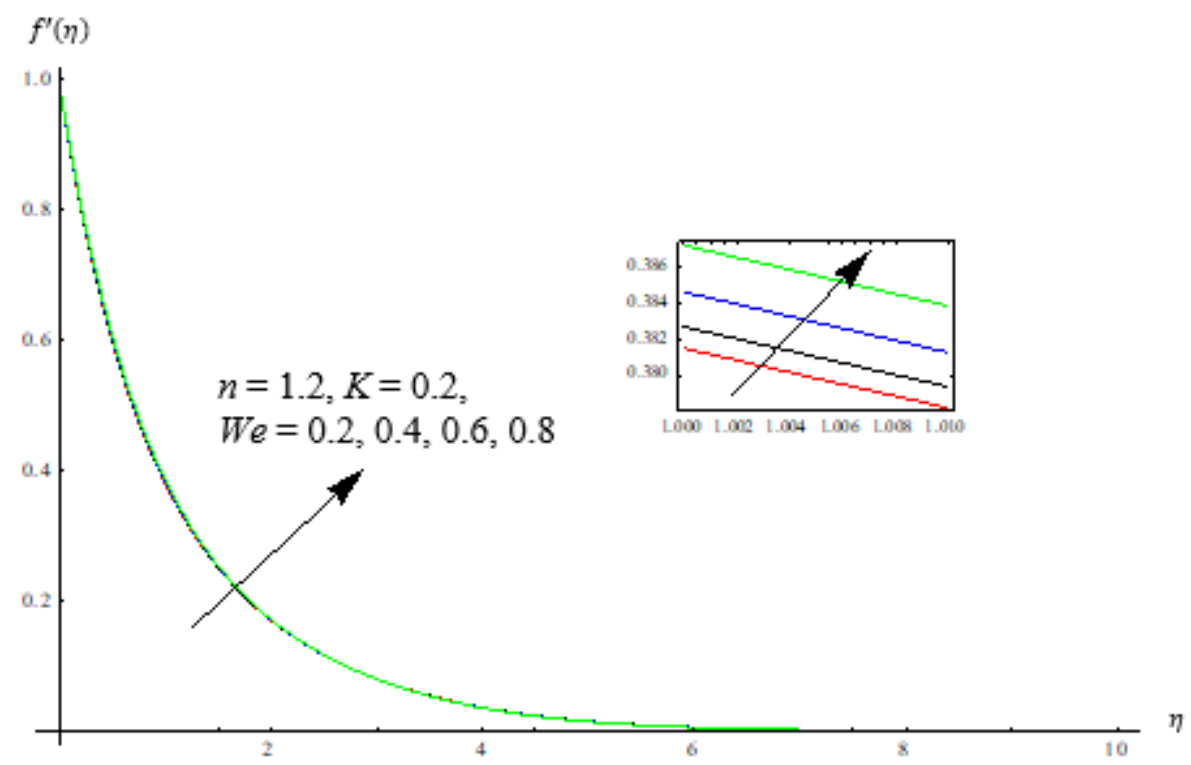

Fig. 5. Velocity profile $f^{\prime}(\eta)$ versus $\eta$ for various values of $W e$ and $n=1.2$

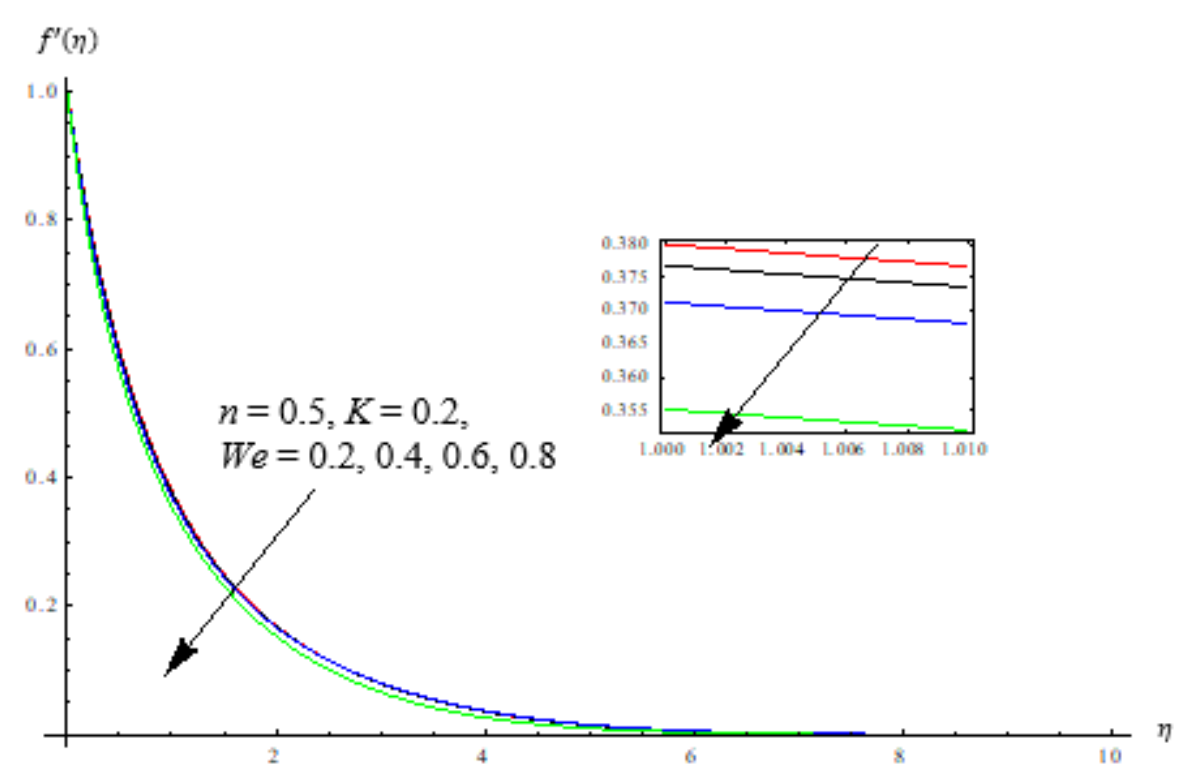

Fig. 6. Velocity profile $f^{\prime}(\eta)$ versus $\eta$ for various values of We and $n=0.5$

\section{Conclusion}

In the present study, a steady Carreau fluid flow over a stretching permeable tube has been considered. Analytical series solutions of the similarity governing equations are obtained by applying the homotopy analysis method. The converging study of the series solutions has been conducted and the results of the present work have been validated by comparing with the existing solutions in the literature. Effects of the power-law index $n$, Weissenberg number We, and 
curvature parameter $K$ on the flow profiles have been examined. From this investigation, we can conclude
i. A conflict behavior happens for $n>1$, the shear-thickening fluid, and $n<1$, the shear- thinning fluid as the Weissenberg number We are increased.
ii. The local skin friction of the fluid is enhanced by the curvature parameter $K$ and Weissenberg number We.
iii. The HAM is appropriate to be used in solving highly non-linear differential equations.

\section{Acknowledgment}

The authors would like to acknowledge the Ministry of Higher Education Malaysia and Research Management Centre-UTM, Universiti Teknologi Malaysia (UTM) for financial support through vote numbers FRGS/1/2019/STG06/UTM/02/22 and 08G33.

\section{References}

[1] Wang, Ch Y. "Fluid flow due to a stretching cylinder." The Physics of fluids 31, no. 3 (1988): 466-468. https://doi.org/10.1063/1.866827

[2] Ishak, Anuar, Roslinda Nazar, and Ioan Pop. "Magnetohydrodynamic (MHD) flow and heat transfer due to a stretching cylinder." Energy Conversion and Management 49, no. $11 \quad$ (2008): 3265-3269. https://doi.org/10.1016/i.enconman.2007.11.013

[3] Ishak, Anuar, Roslinda Nazar, and loan Pop. "Uniform suction/blowing effect on flow and heat transfer due to a stretching cylinder." Applied Mathematical Modelling 32, $\quad$ no. $10 \quad$ (2008): 2059-2066. https://doi.org/10.1016/j.apm.2007.06.036

[4] Lok, Y. Y., J. H. Merkin, and I. Pop. "Mixed convection flow near the axisymmetric stagnation point on a stretching or shrinking cylinder." International journal of thermal sciences 59 (2012): 186-194. https://doi.org/10.1016/j.ijthermalsci.2012.04.008

[5] Rangi, Rekha R., and Naseem Ahmad. "Boundary layer flow past a stretching cylinder and heat transfer with variable thermal conductivity." (2012).

[6] Vajravelu, K., K. V. Prasad, and S. R. Santhi. "Axisymmetric magneto-hydrodynamic (MHD) flow and heat transfer at a non-isothermal stretching cylinder." Applied Mathematics and computation 219, no. 8 (2012): $3993-4005$. https://doi.org/10.1016/i.amc.2012.10.034

[7] Mukhopadhyay, Swati. "Mixed convection boundary layer flow along a stretching cylinder in porous medium." Journal of Petroleum Science and Engineering $96 \quad$ (2012): 73-78. https://doi.org/10.1016/i.petrol.2012.08.006

[8] Mukhopadhyay, Swati. "MHD boundary layer slip flow along a stretching cylinder." Ain Shams Engineering Journal 4, no. 2 (2013): 317-324. https://doi.org/10.1016/j.asej.2012.07.003

[9] Adnan, N.S.M., Som, A.N.M., Arifin, N.M., Bachok, N., Ali, F.M. and Rahim, Y.F. "A Stability Analysis of Boundary Layer Stagnation-Point Slip Flow and Heat Transfer towards a Shrinking/Stretching Cylinder over a Permeable Surface." CFD Letters 12, no. 11, (2020): 97-105. https://doi.org/10.37934/cfdl.12.11.97105

[10] Myers, T. G. "Application of non-Newtonian models to thin film flow." Physical Review E 72, no. 6 (2005): 066302. https://doi.org/10.1103/PhysRevE.72.066302

[11] Usman, A.H., Rano, S.A. and Kumam, P. "Activity of Viscoelastic Nanofluid Film Sprayed on a Stretching Cylinder with Arrhenius Activation Energy and Entropy Generation." Journal of Advanced Research in Micro and Nano Engineering 3, no. 1 (2021): 12-24.

[12] Carreau, Pierre J. "Rheological equations from molecular network theories." Transactions of the Society of Rheology 16, no. 1 (1972): 99-127. https://doi.org/10.1122/1.549276

[13] Tshehla, M. S. "The flow of a Carreau fluid down an incline with a free surface." International Journal of Physical Sciences 6, no. 16 (2011): 3896-3910.

[14] Olajuwon, Ishola Bakai. "Convection heat and mass transfer in a hydromagnetic Carreau fluid past a vertical porous plate in presence of thermal radiation and thermal diffusion." Thermal science 15, no. suppl. 2 (2011): 241-252. https://doi.org/10.2298/TSCI1010260600

[15] Khan, Masood, and Hashim. "Boundary layer flow and heat transfer to Carreau fluid over a nonlinear stretching sheet." AIP Advances 5, no. 10 (2015): 107203. https://doi.org/10.1063/1.4932627 
[16] Hayat, Tasawar, Sadia Asad, Meraj Mustafa, and Ahmed Alsaedi. "Boundary layer flow of Carreau fluid over a convectively heated stretching sheet." Applied Mathematics and Computation 246 (2014): 12-22. https://doi.org/10.1016/i.amc.2014.07.083

[17] Khan, M., M. Irfan, W. A. Khan, and A. S. Alshomrani. "A new modeling for 3D Carreau fluid flow considering nonlinear thermal radiation." Results in physics 7 (2017): 2692-2704. https://doi.org/10.1016/i.rinp.2017.07.024

[18] Khan, Sami Ullah, and Sabir Ali Shehzad. "Electrical MHD Carreau nanofluid over porous oscillatory stretching surface with variable thermal conductivity: applications of thermal extrusion system." Physica A: Statistical Mechanics and its Applications 550 (2020): 124132. https://doi.org/10.1016/i.physa.2020.124132

[19] Abbas, Tariq, Sajid Rehman, Rehan Ali Shah, Muhammad Idrees, and Mubashir Qayyum. "Analysis of MHD Carreau fluid flow over a stretching permeable sheet with variable viscosity and thermal conductivity." Physica A: Statistical Mechanics and its Applications 551 (2020): 124225. https://doi.org/10.1016/i.physa.2020.124225

[20] Al-Azawy, M.G., Kadhim, S.K. and Hameed, A.S. "Newtonian and Non-Newtonian Blood Rheology Inside a Model of Stenosis." CFD Letters 12, no. 11 (2020): 27-36. https://doi.org/10.37934/cfdl.12.11.2736

[21] Salahuddin, T. "Carreau fluid model towards a stretching cylinder: Using Keller box and shooting method." Ain Shams Engineering Journal 11, no. 2 (2020): 495-500. https://doi.org/10.1016/i.asej.2017.03.016

[22] Khan, M. Ijaz, Amit Kumar, T. Hayat, M. Waqas, and Ramayan Singh. "Entropy generation in flow of Carreau nanofluid." Journal of Molecular Liquids 278 (2019): 677-687. https://doi.org/10.1016/j.molliq.2018.12.109

[23] Hayat, T., M. Mustafa, and S. Asghar. "Unsteady flow with heat and mass transfer of a third grade fluid over a stretching surface in the presence of chemical reaction." Nonlinear Analysis: Real World Applications 11, no. 4 (2010): 3186-3197. https://doi.org/10.1016/j.nonrwa.2009.11.012

[24] Liao, Shijun. Beyond perturbation: introduction to the homotopy analysis method. CRC press, 2003.

[25] Liao, Shijun. Homotopy analysis method in nonlinear differential equations. Beijing: Higher education press, 2012. https://doi.org/10.1007/978-3-642-25132-0

[26] Liao, Shi-Jun. "The proposed homotopy analysis technique for the solution of nonlinear problems." PhD diss., Ph. D. Thesis, Shanghai Jiao Tong University, 1992.

[27] Liao, Shijun. "Notes on the homotopy analysis method: some definitions and theorems." Communications in Nonlinear Science and Numerical Simulation 14, no. $4 \quad$ (2009): $983-997$. https://doi.org/10.1016/i.cnsns.2008.04.013

[28] Akbar, N. S., S. Nadeem, Rizwan UI Haq, and Shiwei Ye. "MHD stagnation point flow of Carreau fluid toward a permeable shrinking sheet: Dual solutions, Ain Shams Eng." J 5, no. 4 (2014): 1233-1239. https://doi.org/10.1016/i.asej.2014.05.006

[29] Khan, Masood, and Ali Saleh Alshomrani. "Characteristics of melting heat transfer during flow of Carreau fluid induced by a stretching cylinder." The European Physical Journal E 40, no. 1 (2017): 1-9. 